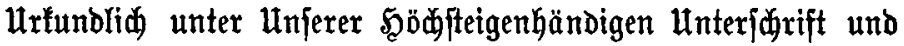

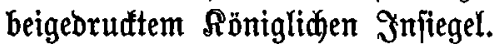

Gegeben Berlin, Den 8. Mai 1867.

(L. S.) Bilgerm.

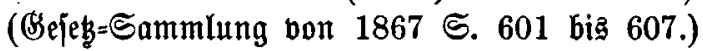

\title{
II. Berarbunugen,
}

betr. die Bezirfe und daz Reffort der Bergbegörden im

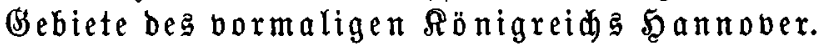

1. Aäninglide Nerorìnung wom 25. Alai 1867 ;

betr. Die Bezirfe und bas Reffort ber Oberbergämter im Bebiete bes vormaligen Rönigreiđgs 5̧annover.

Bir $\mathfrak{B i l h e l m}$, von Bottes \$smaden Rönig von Breutien zc.

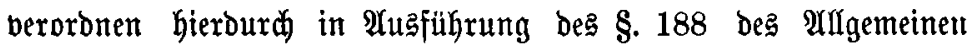
Berggeję̧ę bom 24. Juni 1865 (Gejeg=Sammlung für 1865 Seite 705), jomie ber $\mathfrak{B e r o r o n u n g ~ b o m ~ 8 . ~ M a i ~ b i e j e ̨ ~ J a a h r e s , ~ b e ~}=$

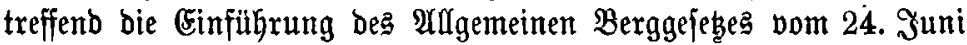
1865 in bas Bebiet bes vormaligen Rönigreidjs Sgannover (Gejeb= Sammlung für 1867 Seite 601), mas folgt:

\section{Irtifel I.}

Der Bezirf dę Dberbergamtę zu Dortmund umfapt bom

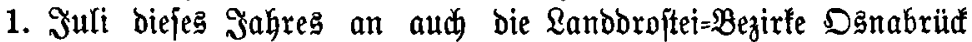

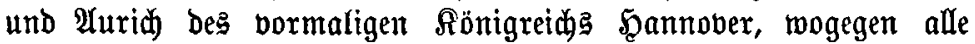
übrigen Sandestheile Dez Ieşteren .bon Dem bezeidinneten Beitpuntte an ben Befinf bes $\mathfrak{B} e r g=$ und Forftamtes zu Elauattal bilden. Dem $\mathfrak{B} e r g=$ und Forftamte $\mathfrak{z}$ (STausthal werben in feiner (sigen= iđaft als Bergbehörbe afle Befugniffe und Dhliegentgeiten eines Sberbergamtes bis auf Beiteres beigelegt.

Qrtifel II.

Mit Dem 1. Juli biejes Jahres find bie Berwaltungen ber Domanial=Bergmerfe, Steinbrübe, Đütten=, Şammerwerłe und $S_{a}=$

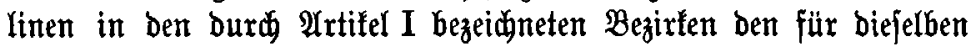
eingeję̧ten Sberbergämtern unterworfen. 
Der Şandelsminifter ift mit ber $\mathfrak{A}$ usfithrung biejer Berord= nung beauftragt.

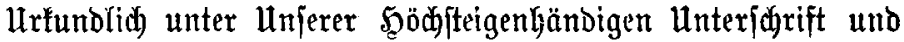

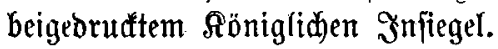

Begeben Berlin, ben 25. Mai 1867.

$$
\text { (L. S.) } \quad \text { Billielm. }
$$

(Gejeb=-Sammlung bon 1867 Seite 735.)

2. Derordnung des ffandelsminiffers vom 1. Zuni 1867 , betr. bie Fefffellung ber Reviere in bem Bebiete bes vormaligen Rönig. reids Şannober.

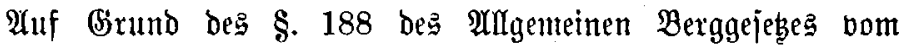
24. Juni 1865 und ber Imerhböbften Beroromung bom 25. Mai

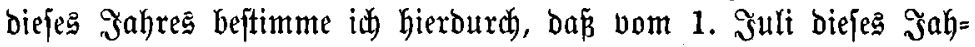
res an

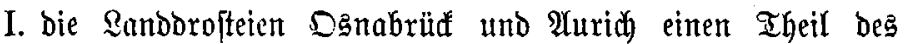
Bergrebieres $\mathfrak{5} \mathfrak{a m} \mathfrak{m}$ an ber Rippe,

II. Die Randoroftei Şildeagheim und die Berghauptmannfafaft Glausthal bą Bergrebier Boslar,

III. bie \&anbdrofteien 5eannober, \&üneburg und Stade bas Bergrebier $5 \mathfrak{a n n o b e r}$ bilben jollen.

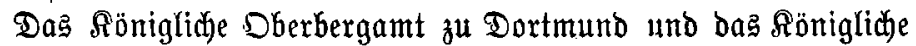

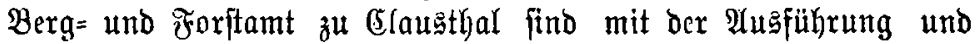
Befanntmadyung biejer Fretftellung Der Bergrebiere burdi bie amtli= Wen Brätter beauftragt.

Berlin ben 1. Juni 1867.

Der Minifter für פandel, Biewerbe und öffentliche $\mathfrak{A} r$ beiten. (gez.) Siraf bon Sţenpliz.

\section{Sekanmtmadjung Deg Berg- und Eorftamts $3 \mathfrak{u}$ Clausthal vom 7. Funi 1867 ,}

betr. bie 2rmafme ber Muthunger.

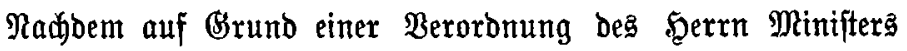
für \$andel, Getwerbe und sffentlidie Urbeiten vom 1. Sunt biefes Sahres 
1. Die \&anboroftei Ђ̧iloeßgheim und bie Berghauptntanmidaft (Slaus= that bas Bergrebier Boslar, mie

2. Die Ranbornfteien Szannover, Lüneburg und Stade bas Berg= rebier $\mathfrak{\$} \mathfrak{n} \mathfrak{n} \mathfrak{D} \mathfrak{b e r}$

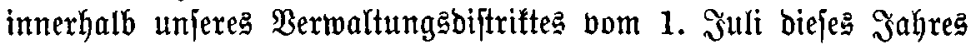
$\mathfrak{a b}$ bilden jollen, und mit $\mathfrak{B a h n n e h m u n g ~ d e r ~ R e v i e r b e a m t e n g e j d a ̈ f ̆ t e ~}$

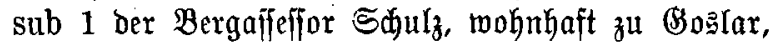

sub 2 ber Bergaffefipr Jung, wohnhaft zu Szannober,

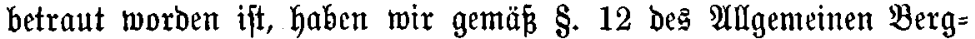
gefebce bom 24. Juni 1865 vorgenannten beiden Revicrbeamten bie $\mathfrak{A} n n a h m e$ Der Muthungen innerbalb Der ihnen übermiejenen

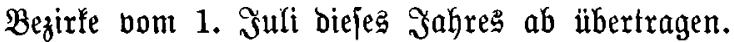

3ur $\mathfrak{H} n=$ und $\mathfrak{A} u f \mathfrak{n}$ ahme oon Muthungen find auf Brand

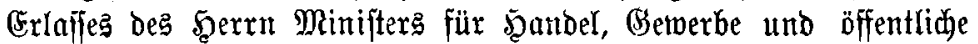
Urbeiten bei ben Mevierbeanten die Stunden bon 8 bis 12 uhr Bormittage und von 3 bis 6 uhr Madjmittags an ben $\mathfrak{B o d h e n t a =}$ gen ausforliep̧lidy beftimmt.

Auser diefen Dienftftunden und auperbalb dę Dienftlofals merben Mutfungen nidgt präfentirt uno protofollariftae Mutfungen nidyt aufgenommen.

$\mathfrak{A} n$ Sonn = und Freiertagen merden protofollarijde Muthungen überbaupt nidgt aufgentommen und bic 倘riftlid eingehenden $\mathfrak{R u}=$ thungen nur während ber 30 ormittagęftunden bon 8 bis $9 \mathrm{lthr}$ präfentirt.

Die Muthangsgejude find nad $\S .13$ bes Berggejeges in zmei gleid)lautenden Eremplaren einzulegen und find ftempelpflidtig.

Die Bermendung bon Preupifinem Stempelpapier ober Stem= pelmarken ift zur Beit now unzuläfftg.

(Slausthal, ben 7. Juni 1867 .

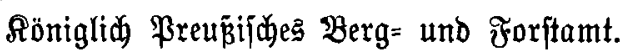

(gez.) v. Sinfingen.

(Staatåanzeiger bon 1867 Seite 2314.) 